\title{
Research Progress of Chimeric RNA and Health
}

\author{
Weikai Chen1, Wei Cui1, Ye Qiu' ${ }^{2 *}$, Di Cui ${ }^{*}$ \\ ${ }^{1}$ Department of Physical Education, Hunan University, Changsha, China \\ ${ }^{2}$ College of Biology, Hunan University, Changsha, China \\ Email: *cuidi616@163.com, *qiuye@hnu.edu.cn
}

How to cite this paper: Chen, W.K., Cui, W., Qiu, Y. and Cui, D. (2021) Research Progress of Chimeric RNA and Health. Health, 13, 454-471.

https://doi.org/10.4236/health.2021.134036

Received: March 12, 2021

Accepted: April 25, 2021

Published: April 28, 2021

Copyright (c) 2021 by author(s) and Scientific Research Publishing Inc. This work is licensed under the Creative Commons Attribution International License (CC BY 4.0).

http://creativecommons.org/licenses/by/4.0/ (c) (i) Open Access

\begin{abstract}
With the development of deep sequencing and bioinformatics technology, a large number of products produced by abnormal RNA splicing, such as chimeric RNA and chimeric/fusion proteins, have been discovered. Natural chimeric/fusion genes are new genes formed by natural fusion of two or more independent genes. Chimeric RNAs can be transcribed by natural chimeric genes, and can also be formed by cis-splicing or trans-splicing of two or more precursor mRNAs. Unlike fusion genes, the production of chimeric RNAs does not involve changes in the DNA level of chromosomes. At first, chimeric RNAs were found as tumor markers. With the deepening of research, researchers also found a large number of chimeric RNAs in normal tissues. From the perspective of biological function, chimeric RNAs can play a biological role in regulating the expression of corresponding maternal genes, translating into chimeric proteins, and forming long non-coding RNAs. The objective of the present study focused on the frontiers of chimeric RNA and reviewed its role in health and tumor study to reveal research progress of chimeric RNA and health and provide a new sight of relative disease treatment. The main conclusion of this review is that chimeric RNA may serve as a biomarker for specific tumor diagnose and treatment while its role in normal physiology needs to be revealed.
\end{abstract}

\section{Keywords}

Chimeric RNA, Fusion Gene, Sis-Splicing, Trans-Splicing, Tumor, Health

\section{Introduction}

Chimeric or fusion gene refers to the DNA sequence formed by two or more independent genes through chromosome recombination, read-through transcription of adjacent genes, trans-splicing, etc. Chromosome recombination events include chromosome deletion, duplication, insertion, inversion, translocation 
and annular chromosomes, etc. All of these mutations can form chimeric genes [1]. Fusion gene was firstly reported in 1960 by Peter Nowell et al. [2]. In 1973, Janet Rowley discovered the first chimeric gene formed by chromosomal translocation (BCR-ABL1) in chronic myelogenous leukemia (CML) [3]. Later, with the deepening of the research, a growing number of chimeric genes have been found in tumor formation such as chronic myeloid leukemia (BCR-ABL1) [3], acute lymphocytic leukemia (AML1-ETO) [4], acute promyelocytic leukemia cells (AML1-ETO) [5], striated muscle tumor (PAX3-FOXO1) [6], prostate (TMPRSS2-ERG) [7], serous ovarian cancer (BCAM-AKT2) [8], etc. Concept of chimeric RNA and synchronous chimeric gene, is regarded as a product of chimeric gene transcriptional expression, namely from different gene exon fragments chimeric transcript, and the follow-up study confirmed that the chimeric RNA could also be formed by two or more than two independent precursor mRNA (pre-mRNA) conveniently cis-splicing or by trans-splice. More and more chimeric RNAs are being discovered continuously, and they are collected in several large databases including HYBRIDdb [9], FusionCancer [10], ConjoinG [11], TICdb [12], dbCRID [13], FusionDB [14], ChiTaRs [15], ChimerDB [16], Mitelman [17].

With the systematic deepening of research on chimeric RNA, a large number of chimeric RNAs have been detected in tumor and non-cancer tissues respectively, which has become a new hotspot of transcriptomics research. The study on its formation characteristics and function is conducive to our comprehensive understanding of the composition and function of the genome [18]. In this paper, the formation mechanism, expression characteristics and biological functions of chimeric RNAs were reviewed, aiming to comprehensively understand the research situation and development trend of chimeric RNAs, and to provide non-genetically modified chimeric RNAs intervention strategies and ideas for the diagnosis and treatment of clinically related diseases.

\section{The Formation Mechanism of Chimeric RNA}

Gene refers to a DNA fragment with genetic information, and a fusion gene refers to the fusion of two genes in whole or in part to form a new gene, which is the result of chromosome recombination, deletion or chromosome inversion, and the product of transcription is chimeric RNA. Chimeric RNA is a mosaic of two transcripts. It can be the product of fusion gene expression, or it can be obtained by further splicing after gene transcription. It is a non-collinear RNA editing and does not involve chromosome change. The splicing mechanisms that have been reported include cis-splicing and trans-splicing. Of course, there may be other unknown non-splicing mechanisms, and further research is needed (Figure 1).

\subsection{Overview of Genes and RNA}

Genes are fragments of DNA that have genetic effects, and their expression is 

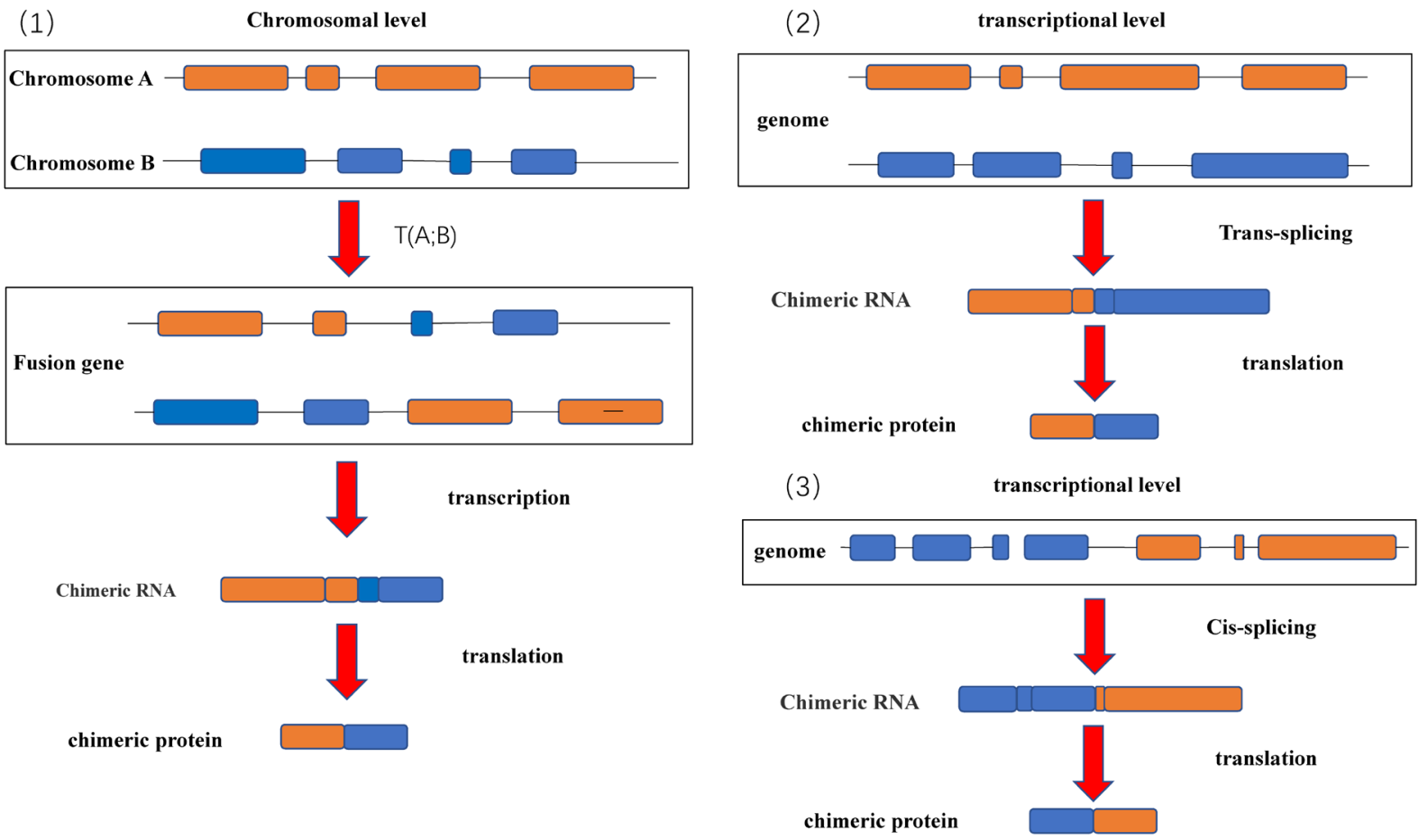

Figure 1. Pattern diagram of chimeric RNA production pathway.

realized by DNA controlling the synthesis of proteins. Chromosome is the carrier of gene, its structure is stable, is the basis of cell regular division and proliferation. However, heredity is relative, variation is absolute [19]. DNA molecular DNA nucleotide sequence determines the messenger RNA in the order of the ribonucleotide, messenger RNA in the order of the ribonucleotide and determines the amino acid sequence, amino acid sequence ultimately determines the specificity of the structure and function of protein, making organisms exhibit various genetic traits. In recent 10 years, one of the most popular studies in the field of biomedical science is the study of RNA, which covers from miRNA to other short non-coding RNA (including siRNA, miRNA, piRNA), long non-coding RNA (incRNA), fusion RNA (chermicRNA), cirRNA (cirRNA), and non-coding RNA has become the focus of research. Non-coding RNAs refer to RNAs that do not encode proteins, including RNAs with known functions such as rRNA, tRNA, snRNA, snoRNA and microRNA, as well as RNAs with unknown functions. The two common non-coding RNAs, rRNA and tRNA, are the most abundant in cells. It is estimated that at least one-third of human genes are regulated by non-coding RNAs.

\subsection{Discrimination of the Concepts of Fusion and Chimerism}

Fusion gene refers to two or more genes coding region of end to end, in the same set of regulatory sequence (including promoter, enhancer, ribosome binding sequence, termination, etc.) under the control of its parent gene can be located on different chromosomes, different strands of DNA can also be located on the same chromosome, or on a single strand of DNA, to produce a chimeric gene 
was absent or chromosome inversion of chromosome translocation and results. Chimeric genes can connect the functional domains of genes from different sources, combine new functions, change the characteristics of the original molecules, and have specific biological functions. However, the different exon sequence between chimeric genes and their parent genes may be one of the reasons for the disorder of the inherent balance system. The transcriptional product of chimeric genes is chimeric RNA, which can be translated into chimeric proteins. The DNA sequences formed by chromosomal recombination are chimeric/fusion genes. Chromosome deletion, duplication, insertion, inversion, translocation and ring chromosomes can form chimeric genes, and their transcriptional intermediates can form chimeric RNA. Chimeric RNA is the product of fusion gene expression, can not only through the chromosome translocation, missing or chromosome inversion approach to form, can also through the cis adjacent gene splicing and trans splicing methods such as form, but trans splicing is the main way to generate Chimeric RNA, also is a kind of important eukaryotic cell RNA transcription after processing mechanism, and the corresponding variety of RNA isomer, make more than one gene can be translated into a variety of skin sequence, become the important manifestation of diversity of gene expression [20]. The formation of chimeric RNA is an important link in the regulation of gene expression in eukaryotes. It plays a biological role by regulating the expression of corresponding parent genes or forming chimeric proteins, and directly affects the coding information of corresponding genes.

\subsection{Chimeric/Fusion Genes Are Transcribed to Form Chimeric RNA}

$\mathrm{BCR}-\mathrm{ABL}$ is about $8.5 \mathrm{~KB}$ mRNA transcription product for long, namely the chimeric RNA, the combination of translation product for $210 \mathrm{kDa}$ protein, including 1104 amino acid residues and BCR ABL encoding 920 amino acid residues, have stronger tyrosine protein kinase activity, promote cell proliferation, apoptosis, is CML oncogene, depending on the gene fracture zone BCR-ABL chimeric genes and their chimeric RNA, chimeric protein products also shows diversity [21]. From the perspective of gene structure, BCR-ABL chimeric gene is a typical domain fusion of heterologous genes, encoding carcinogenic functional proteins. Similar to BCR-ABL, the chimeric gene tel-AML1 is specifically expressed in approximately $25 \%$ of childhood Acute lymphoblastic leukemia (ALL), i.e., T $(12 ; 21)$ (p13; q22) translocation, its mother genes TEL and AML1 are classical transcription factors, involved in regulating the transcription of target genes related to cell differentiation and proliferation, and the latter is mainly expressed in hematopoietic tissue and involved in regulating the expression of genes related to hematopoietic cell development.Tel-AML1 is transcribed into chimeric RNA and translated to form chimeric proteins, which competitively inhibit the transcription activity of maternal genes. In Acute myeloid leukemia (AML), T $(8 ; 21)$ (q22; q22) Chromosome translocation resulted in the forma- 
tion of chimeric/fusion gene AML1-ETO, and the transcriptional chimeric RNA produced chimeric proteins, which mainly acted on the over activation of other genes to affect hematopoietic differentiation, proliferation and apoptosis and other functions, and its downstream regulatory proteins included SIRT1, P53 and EZH1. Rhabdomyosarcoma (RMS) is a common soft tissue cancer in which T (2; 13) (q35; q14) chromosomal translocation resulted in PAX3-FoxO1 or T $(1 ; 13)$ (p36; q14) Translocation resulted in the specific expression of PAX7-FoxO1 chimeric genes, and the translation of transcriptional chimeric RNA into chimeric protein not only affected the myogenic regulation and differentiation of parent genes PAX3 and FoxO1, but also affected the expression of downstream target molecules including P16INK4A and P57Kip2 or their activity to mediate muscle cell canceration. These gene fuses occur on different chromosomes, and in fact they may occur on the same chromosome. 50\% of Prostate cancer adenocarcinoma (PCA) patients contain TMPRSS2-ERG chimeric gene, are the chromosome $21 \mathrm{q} 22.2$ and $21 \mathrm{q} 22.3$ sequence missing or balanced translocation gene fusion results, through the transcription of chimeric RNA eventually chimeric protein formation, the positive feedback to promote female highly expressed genes ERG, which involved in embryonic development, cell proliferation, differentiation, angiogenesis, inflammation, and apoptosis related molecules regulate and promote the development of tumor, is an important one of the main causes of deterioration of PCA [22]. In addition, about 7\% of High grade serous ovarian cancer (HGSC) had BCAM-AKT2 gene fusion, i.e., T (19; 19) (q13; Q13) In frame fusion, its chimeric RNA translated chimeric protein products to play kinase activity and connect with the biofilm, continuously activating the AKT2 kinase-mediated PI3K/Akt signaling pathway to promote cancer. Chimeric RNA produced by gene fusion transcription often plays a biological role by producing chimeric proteins and is closely associated with the majority of soft tissue tumors. In laboratory conditions, the study of fusion genes tends to be more extensive at the RNA level. On the one hand, after the occurrence of gene fusion event, even though the breakpoint generally occurs in the intron fragment, the function is usually generated by chimeric RNA or chimeric protein, and the only way for the latter is chimeric RNA. Transcriptome sequencing is more popular in the study of chimeric RNA due to its high efficiency and low cost. On the other hand, the exon spliced transcriptome sequences obtained by general sequencing are easier to recognize the chimeric sequences, and the amino acid sequence of the chimeric protein of the translated product can be determined according to the chimeric RNA sequences to design antibodies. The disadvantage of using chimeric RNA to study fusion genes is that if gene fusion does not produce new transcripts, i.e. fire fusion, the results cannot be detected at the chimeric RNA level, and only the corresponding results can be obtained by sequencing at the DNA level. Therefore, chimeric RNA produced by gene fusion is often recognized as a tumor-related marker and also used for the early diagnosis of some cancers. With the deepening of research, there are also targeted drug 
research and development aimed at the level of carcinogenic chimeric RNA, which provides a non-genetic genetic programming clinical intervention scheme for the diagnosis and treatment of related diseases.

\subsection{Chimeric RNA Cis-Splicing Mechanism}

Cis-splicing occurs in adjacent genes on the same DNA strand, and the exons of the transcription product are combined with each other to form a chimeric RNA. Some scholars Read-through chimera. In recent years, scholars have discovered many chimeric RNAs in which the exons of adjacent genes are chimeric with each other through a large number of paired-end RNA-Seq data studies. [23] [24], Qin detected more than 300 chimeric RNAs in prostate cancer and non-cancer samples, of which $30 \%$ were cis-spliced chimeric RNAs [25]. SLC45A3-ELK4 has been extensively studied as a potential prostate cancer biomarker [26] [27], The maternal gene of this mosaic is located on chromosome 1q32, use Fluorescence in situ hybridization, Column comparison genomic hybridization, Southern blot, qPCR. The exons of the two genes, SLC45A3 and ELK4 were analyzed. The results of the analysis showed that there was no interstitial DNA deletion between the exons of these two genes. In the absence of corresponding chromosomal recombination, SLC45A3-ELK4 chimeric fusion transcription in prostate cancer cells is produced by cis-splicing adjacent genes instead of trans-splicing [28] [29]. Through the current research on the ligation sites of known chimeric RNAs, it is found that there are no specific sequence templates around the chimeric ligation sites, which also reflects the limitations of current case studies of cis-splicing. As more and more cis-spliced chimeric RNAs are continuously discovered, the identification of their gene splicing will also be a difficult problem for future research.

\subsection{Chimeric RNA Trans-Splicing Mechanism}

Trans-splicing occurs when one or more exons of two independent RNA transcripts are combined with each other to form a chimeric RNA, trans-splicing. It was first discovered in the study of trypanosomes [30]. Then in tobacco [31] and chlamydomonas [32]. Also confirmed the existence of trans-splicing. However, its formation mechanism is not yet clear, and it may be related to the transcription and splicing of a specific sequence or the closeness of the spatial structure [33]. In the 1990s, people began to discover the existence of trans-splicing in mammals [34]. RNA splicing is a key step to produce mature mRNA. RNA trans-splicing combines two independent pre-mRNA molecules, and pre-mRNA molecules may originate from different genes [35]. Or form a chimeric nonlinear RNA in the same gene that may have a different function from the original molecule [36]. Trans-spliced RNA can encode a new protein or be a non-coding or regulatory RNA. These new RNAs not only increase the complexity of the proteome, but also provide a new regulatory mechanism for gene expression. More and more evidences show that trans-splicing often occurs in cell physiolo- 
gy and pathological processes. In addition, mRNA reprogramming based on trans-splicing has been successfully applied to RNA-based treatment of human genetic diseases [37].

\section{Physiological Function and Clinical Value of Chimeric RNA}

\subsection{Regulation of Maternal Gene Expression by Chimeric RNA}

Chimeric RNA itself can be used as a clinical diagnostic marker and drug targeting for some tumors. The BCR-ABL gene can be expressed in the leukemia cells of about $95 \%$ of patients with chronic myelogenous leukemia and $20 \%$ of patients with acute lymphoblastic leukemia, so the BCR-ABL gene can be used as a molecular diagnostic marker for leukemia [38]. In 2007, Soda et al. found EML4-ALK chimeric RNA in the cells of a male lung cancer patient [39]. His clinical features are clearly different from those of patients with epidermal growth factor receptor positive, and EML4-ALK can be detected in most non-smokers of non-small cell lung cancer (NSCLC) patients, which has unique pathological features [40]. Afterwards, inhibitors targeting EML4-ALK chimeric RNA continued to appear, which greatly improved the quality of life and treatment of patients with non-small cell lung cancer, including crizotinib, ceritinib and alectinib appeared and other inhibitors. The EML4-ALK fusion gene, as a tumorigenic variant gene discovered in recent years, has become a new target for the clinical treatment of advanced NSCLC due to its unique clinic pathological characteristics and relatively high positive rate [41]. Guo, Qi and others have confirmed through research that the TMPRSS2-ERG fusion gene found in prostate cancer is a specific genetic change of prostate cancer, and found that TMPRSS2-ERG and ERG proteins are not found in normal prostate glands and normal glands adjacent to cancer. Show that it can be used as a specific marker for the clinical diagnosis of prostate tumors [42]. In the study of high-grade serous ovarian cancer (HGSC), Kannan et al. showed that BCAM-AKT2 expression is a new mechanism for AKT2 kinase activation in HGSC. BCAM-AKT2 is the only fusion gene in HGSC that has been proven to be able to translate aberrant kinase fusion proteins with oncogenic properties. This recurring genomic change is a potential therapeutic target of HGSC and a marker of clinically relevant subtypes [8].

\subsection{Function of Chimeric Proteins Translated from Chimeric RNA}

Chimeric RNA forms a long chain of non-coding RNA, and a large number of chimeras may have the function of non-coding RNA. Supporting this view is a recent study that identified more than 58,000 transcripts as long non-coding RNA [43]. Qin found in prostate cancer research that fusion transcription can function as a long non-coding chimeric RNA [44]. In SLC45A3-ELK4 chimeric RNA, the protein encoded by chimeric RNA is the same as ELK4 [45]. When the chimeric RNA (instead of ELK4) is silenced, the cell proliferation of androgen 
dependent prostate cancer cells and castration-resistant prostate cancer cells are inhibited. This growth arrest can be rescued by a fusion of exogenous expression and a mutant designed to prevent the translation of the ELK4 protein. Under the same conditions, the mutant can also inhibit several other targets of CDKN1A and SLC45A3-ELK4. Taken together, these results indicate that the chimeric RNA SLC45A3-ELK4 regulates the proliferation of cancer cells through its transcript rather than a translated protein.

Chimeric RNA can produce chimeric protein. By connecting the functional domains of different genes, Chimeric protein can change the function of parent genes, that is, change the function of pre-fusion original genes, increase the diversity and complexity of transcriptome and proteome, block the normal signaling pathway, and initiate or activate the growth of cancer cells. Chimeric RNA consists of two or more exons of different genes and has the potential to encode new proteins that change the phenotype of cells [46]. Researchers have discovered PAX3-FOXO1 chimeric RNA in rhabdomyosarcoma. The chimeric RNA produces a new PAX3-FOXO1 chimeric protein from PAX3 and FOXO1. This protein has greater in vitro and in vivo transcriptional activity than wild-type PAX3. The PAX3-FOXO1 protein of fibroblasts and rhabdomyosarcoma cell lines can induce simultaneous myogenesis and prevent them from differentiating into mature muscles. JAZF1-JJAZ1 chimeric RNA produces a new chimeric protein, which plays an important role in regulating cell proliferation and survival [22]. CTBS-GNG5 encodes a new protein that can regulate cell proliferation and migration [47]. The BCR-ABL chimera found in leukemia produces p210-encoded protein, which promotes the level of tyrosine kinase, causing cells to lose their adaptability to the surrounding environment and inhibiting the rate of cell apoptosis [48]. When investigating the expression of 32 recurrent cancer chimeric RNA in esophageal squamous cell carcinoma patients and cancer cell lines, Zhang $\mathrm{H}$ et al. found that GOLM1-MAK10 is a highly enriched cancer chimeric RNA in ESCC, demonstrating that the chimeric GOLM1-MAK10 encodes a secreted chimeric protein [49].

\subsection{Chimeric RNA Is Recognized as Non-Coding RNA}

SOAP fuse software is used by Tang Yue in infant brain tissue RNA dorsolateral prefrontal cortex cells-seq data analysis, and they verified the 599 chimeric RNA expression, and INTERCHR INTRACHR-SS-zero gap and INTRACHR-OTHER three chimeric types of proportion is $46 \%, 51 \%$ and $43 \%$, of which high express DUS4L-BCAP29 chimeric RNA is widely expressed in normal tissues, cells, and participate in cell proliferation, and OTHER functions. It is suggested that DUS4L-BCAP29 is not suitable as a tumor marker for gastric cancer and prostate cancer, and may play a role in the form of non-coding RNA, which may be related to the phosphorylation inhibition of STAT3 [50]. With the development of deep sequencing, transcriptomics and bioinformatics technology, a large number of products indirectly produced by RNA abnormalities, namely chimeric RNA and fusion proteins, have been found. Unlike fusion genes, chimeric 
RNA production does not involve changes in the DNA level of chromosomes. Ke et al. found that dendritic cell-derived exosomes can load tumor characteristic chimeric RNA, which may provide a new therapeutic strategy for the research and development of exosomal tumor vaccines [51]. Natural chimeric gene is a new gene formed by the natural fusion of two or more independent genes. The discovery of this type of gene breaks through the classical understanding of "one gene corresponds to one stain" and expands the concept of gene. In the process of human cancer research, the combination of many genes can result in tumor related diseases, as a marker of cancer molecular diagnosis based on the research concerning the chimeric gene of bioinformatics, with cancer gene as the breakthrough point, from natural characteristics, transcription, adjust the chimeric gene fusion, and the structure of the fusion protein domain combination form and function, etc., combining the research group of related work, summarized the research progress on the structure and function of the chimeric gene fusion, discusses the difficulties and challenges, the current research work and the Mosaic law application in design of the new gene were discussed.

\section{Chimeric RNA in Tumors}

Chimeric RNA plays an important role in the diagnosis and treatment of tumors. Most research on RNA chimera has focused on its recognition and functional characterization in malignant cells. Its long-term goal is to improve the level of cancer diagnosis and enhance the understanding of tumorigenesis. The most famous cancer chimeric RNA BCR-ABL1, which encodes a tyrosine kinase, which has a constitutive activity in cancer cells, leading to increased proliferation of hematopoietic stem cells [52] [53]. BCR-ABL1 is the target of an effective anticancer drug called Gleevec [54]. EML4-AKL found in non-small cell lung cancer can improve the prognosis after targeted treatment with ALK inhibitors [22]. D2HGDH-GAL3ST2 is located in prostate cancer. It is more easily detected in advanced cancers, indicating that it plays an important role in the development of cancer. Consistently, silencing this fusion leads to a significant reduction in cell proliferation and cell motility. SLC45A3-ELK4 is specific to prostate cancer cells. When the gene is silenced, it inhibits cell growth by inducing cell cycle arrest in the G1 phase [62]. The silencing of another recurring chimera, SLC2A11-MIF, leads to cell cycle arrest and cell proliferation reduction. Therefore, it is believed that the chimeric fusion RNA produced by intergenic splicing represents a new biomarker and/or therapeutic target, chimeric RNA Proven to be an ideal cancer biomarker and drug target [55].

Chimeric genes have good application prospect and important research value in medicine. They can be used as molecular markers for clinical diagnosis of cell carcinogenesis. For example, many chimeric genes that can serve as molecular diagnostic markers have been found in diseased tissues such as soft tissue tumors, breast cancer, epithelial cancer, prostate cancer, chronic myelogenous leukemia, acute leukemia, and non-small cell lung cancer. 


\subsection{Chimerism between Soft Tissue Tumor and EWS Gene}

Soft tissue tumors refer to tumor-like hyperplasia of non-epithelial or mesenchymal tissues, such as smooth muscle, nerve sheath, skeletal muscle, lipid membrane, endothelium and fibroblasts. Soft tissue tumors are caused by chromosomal translocation. Relative to cancer, in the development process and its mechanism has certain difference, does not appear from benign to malignant to transfer the multi-stage process, the development of cancer is composed of multiple cancer gene mutation or the result of the interaction, and tumor suppressor genes and tumor is caused by a single genetic defect, such as lack of chromosome translocation, etc. [22].

\subsection{C-erbB-2 Chimeric Gene and Breast Cancer}

By studying the correlation of c-erbB-2 expression in breast cancer, Li et al. [56] concluded that c-erbB-2 was a cell-derived oncogene, which is normally inactive and involved in the regulation of cell growth and differentiation. When activated by some factors in vivo and in vitro, c-erbB-2 has the activity of transforming into tumor. Currently, c-erbB-2 has been recognized as an oncogene involved in the occurrence and progression of breast cancer. Overexpression of c-erbB-2 often indicates high degree of malignancy and poor prognosis, which reflects that the occurrence and progression of breast cancer is the result of multiple gene mutations and synergistic accumulation.

\subsection{BCR-ABL1 Fusion Gene and Chronic Myelogenous Leukemia}

Chronic myeloid leukemia is a kind of hematopoietic stem cell cloning proliferative diseases, also known as slow each cell leukemia, or CML1, it is the result of the interactive translocation chromosomes generate chimeric oncogene, in normal cells, ABL1 encoding protein $\mathrm{N}$ end contains a inhibit tyrosine kinase activity area, to the active control. When BCR is fused with ABL1, the kinase inhibitory region of $\mathrm{ABL} 1$ is inactivated, the molecular conformation is changed, and the kinase activity is increased abnormally, which activates many proteins and enzymes that regulate the cell cycle, and accelerates cell division, leading to the occurrence of canceration. Its molecular mechanism is no. 22 dyeing genomic chronic myelogenous leukemia (orange) and chromosome 9 (blue) have taken place in the fracture, and fracture may lessen was born in the interior of the BCR gene and ABL1, lead to two broken in half, in the process of genome reassemble, key exception happens, namely the BCR gene first half and half after ABL1 gene splicing into a new, under normal circumstances should not exist BCR-AB1L genes. Studies have found that BCR-ABL chimeric RNA can produce new chimeric protein, the chimeric protein has enhanced the role of tyrosine kinase activity, cause cells to a variety of protein tyrosine phosphorylation level and the function of the cell microfilament motor protein changes, thus disrupting normal signal pathways inside the cell, the cell lose reactivity on the surrounding environment, thus inhibiting the occurrence of apoptosis. 


\subsection{BCR-ABL1 Chimeric Gene and Epilepsy}

Some scholars found that the bcr-abll chimeric gene function defect in patients with severe epilepsy, genetic testing is diagnosed on chromosome 22 and 9 caused by unbalanced translocation, namely human chromosome 22 long arm section on chromosome translocation to nine long arm, formed a small new chromosome 22, 22 chromosome translocation to the breaking point of regional clusters BCR and chromosome 9 ABL1 fusion, form the bcr-abll chimeric gene. But the cousin body beside the patient test, did not suffer from epilepsy, has the problem of balanced chromosomal translocation, follow-up investigation, cousin happens in order to avoid a genetic defect, the prenatal genetic diagnosis, normal first child birth, but after every pregnancy test has problems of chromosome translocation fetus, abortion, only show announcement shows everything is normal, until the inspection company to prepare pregnant, the result was born with a chromosome translocation of birth defects in newborns, subsequently, the parents will be inspection company to court, in the end, was sentenced to compensate the plaintiff and neonatal tens of millions of dollars. This event indicates that the cousin with balanced chromosomal translocation may give birth to a newborn with chromosomal translocation, although he does not show abnormal gene function himself [57].

\section{Chimeric RNA in Normal Non-Cancer Cells}

Gene fusion and its products (chimeric RNA and chimeric protein) were once considered to be unique features of cancer, however, chimeric RNA can also be found in normal cells. They play an important role in normal cell physiology, and their dysregulation can induce tumorigenesis and affect cell differentiation [58]. The researchers found that the trans-spliced JAZF1-JJAZ1 mRNA can also be detected in normal endometrial stromal cells, indicating that the fusion gene product is not limited to cancer or precancerous cells. Among them, JAZF1-JJAZ1 mRNA and its encoded protein were basically as same as the protein synthesized by chimeric RNA in tumor cells. In cultured cells, the chimeric protein has anti-apoptotic properties. When the unrecombined JJAZ1 allele is silenced, the chimeric protein can promote proliferation [59]. As shown in some cases, the same mosaics seen in cancer can also be found in samples of non-malignant tumors. Although some of these chimeras may be early indicators of this disease, recent evidence suggests that chimeric RNA, as a phenomenon, is not limited to cancer. In rhabdomyosarcoma (RMS), a specific chromosome is easily affected. Position t $(2 ; 13)$ (q35; q14) leads to chimeric oncogenic transcription factor PAX3-FOXO1, which has been identified as a marker of RMS aggressive alveolar subtype. But during normal muscle cell differentiation, this chimeric RNA was detected at the moment when no chromosomal translocation was observed. The same chimeric RNA was also detected in muscle biopsy. These observations sounded the alarm for the use of this chimeric RNA as a biomarker, especially for monitoring related diseases [60]. Some 
scholars have also found that the DUS4L-BCAP29 fusion transcript exists in a variety of normal tissues. It also exists in non-cancer epithelial cells and fibroblasts. In terms of quantification, the fusion transcription is found in non-cancerous, gastric and prostate cell lines and tissues. The expression is comparable to that in cancer cell lines and tissues. Overexpression of DUS4L-BCAP29 can promote cell growth and movement, even in non-cancerous cells. Therefore, DUS4L-BCAP29 cannot be used as a cancer biomarker. On the contrary, it is a fusion transcript that exists in normal physiology, and its growth-promoting effect is not unique to cancer cells [61]. Recently, the DUS4L-BCAP29 chimeric RNA was found in the brain tissue of infants and young children. It is not only involved in mediating basic physiological functions such as the proliferation, cycle and apoptosis of human umbilical cord mesenchymal stem cells (Huc-MSCs), but it may also be Strand-encoding RNA forms play a role [62].

\section{Application of Chimeric RNA as a Drug Target}

RNA interference tests have shown that some chimeric genes can promote the growth of cancer cells. In view of the special connection between chimeric genes and cell canceration, some studies have used chimeric RNA as drug targets for the treatment of cancer. For example: PAX3-FoxO1 chimeric RNA and rhabdomyosarcoma, SLC45A3-ELK4 chimeric RNA and prostate cancer, TBLR1-RARA chimeric RNA and acute promyelocytic leukemia, RUNX1-ETO fusion protein and acute myeloid leukemia, and EML4-ALK and non-small cell lung cancer, etc.

\subsection{PAX3-Fox01 Chimeric RNA and Rhabdomyosarcoma}

All kinds of mechanism are different, but the common differentiation of skeletal muscle and nerve tumors, and the results show that the skeletal muscle and nerve phenotype gene expression, with $10 \%$ of acini leiomyosarcoma has the dual phenotype, leiomyosarcoma is originated in the striated muscle cells or the striated muscle cell differentiation of mesenchymal cells of a malignant tumor, it is one of the most common cancer in children and adolescents. Acinar rhabdomyosarcoma is caused by $\mathrm{T}(2 ; 13)$ The chromosomal translocation leads to the chimeric PAX3-FoxO1 gene. This chimeric RNA has greater transcriptional activity in vivo and in vitro than wild-type PAX3, and is currently used as a standard diagnostic variable for the diagnosis of acinar rhabdomyosarcoma. Yuan $\mathrm{H}$ et al. found that chimeric fusion RNA PAX3-FoxO1 associated with acinolar rhabdomyosarcoma was also present in normal non-cancer cells and tissues, as well as in aborted fetuses and during muscle differentiation of stem cells.

\subsection{SLC45A3-ELK4 Chimeric RNA and Prostate Cancer}

Rickman DS, etc. in prostate cancer research found that produced by SLC45A3 gene and gene fusion ELK4 SLC45A3-ELK4 chimeric RNA detected in prostate cancer cells not only, also was found in normal prostate tissue, results proved that the chimeric RNA itself not only can be used as a clinical diagnosis of tumor 
markers and drug targeting, and can express its biological function in normal non-cancerous cells [63].

\subsection{TBLR1-RARA Chimeric RNA and Acute Promyelocytic Leukemia}

Some researchers found that TBLR1-RARA is a novel chimeric RNA in acute promyelocytic leukemia, and it was derived from a $\mathrm{T}(3 ; 17)(\mathrm{q} 26 ; \mathrm{q} 21)$, the histone deacetylase inhibitor cidabamine can significantly inhibit the proliferation of acute promyeloid leukemia cells by inducing apoptosis and promoting cell differentiation through cycle arrest, providing a new idea for the treatment of acute promyeloid leukemia.

\subsection{Chimeric RUNX1-ETO Protein and Acute Myeloid Leukemia}

The RUNX1-ETO fusion protein was found in acute myeloid leukemia. RUNX1 is a key transcription factor for hematopoietic stem cell differentiation. RUNX1 contains a DNA-bound RUNT homologous domain, and ETO is a transcriptional repressor. The RUNX1-ETO chimeric protein not only retains the DNA binding domain of RUNX1, but also inherits the ability of RUNX1 to bind the regulatory region of the target gene promoter, and also contains most of the domain of the transcription repressor ETO. The chimeric protein is competitive with the parent gene binding target gene, inhibits the transcription of the parent gene, interferes with the expression of its normal function, and blocks differentiation to induce acute myeloid leukemia [64] [65].

\section{Problems and Prospects}

With the advancement of the medical level, people are constantly exploring the chimeric RNA. The mechanism of chimeric RNA production and the chimeric RNA formed by other unknown mechanisms will help discover new biomarkers and drugs for the treatment of diseases. Chimeric RNA not only exists in cancer genes, but also in normal genes. With the discovery of more and more tumor driver genes, people have a deeper understanding of tumor occurrence and development. At the same time, more targeted therapy drugs also bring hope to the majority of cancer patients. At present, chimeric RNA is increasingly being valued by scientists, and more and more experiments are put into the ranks of chimeric RNA research. With the development of RNA sequencing technology and molecular biology, there are still greater challenges waiting for humans to face and overcome, such as the problem of drug resistance, the detection of mutant genes, the discovery of unknown mutations, and more targeted detection methods, and to clarify the characteristics of the chimeric RNA signaling pathway. In the future, new sequencing technologies, especially full-length sequencing and the development of new software, will help discover more chimeric RNAs. Many chimeric RNAs discovered so far have not been confirmed to be gene fusions or cancer-specific products. Therefore, before we define chimeric 
RNAs as cancer markers, we need to verify a sufficient number of non-cancer control samples. One of the bottlenecks is the lack of high-throughput methods to study the functions of chimeric RNAs, the formation mechanism of non-traditional chimeric RNAs and their relationship with gene fusion are also questions that urgently need answers. I believe that with the development of science and technology, these problems will be solved, and humans will have a more thorough understanding of chimeric RNA and contribute to people's healthy life in the future.

\section{Conflicts of Interest}

The authors declare no conflicts of interest regarding the publication of this paper.

\section{References}

[1] Li, Y.X., Zhang, T.T. and Ma, L. (2018) Structural Characteristics of Natural Chimeric Genes and Their Implications for Gene Design. Genetic, 40, 135-144.

[2] Nowell, P.C. (1962) The Minute Chromosome (Phl) in Chronic Granulocytic Leukemia. Blut, 8, 65-66. https://doi.org/10.1007/BF01630378

[3] Rowley, J.D. (1973) Letter: A New Consistent Chromosomal Abnormality in Chronic Myelogenous Leukaemia Identified by Quinacrine Fluorescence and Giemsa Staining. Nature, 243, 290-293. https://doi.org/10.1038/243290a0

[4] Romana, S.P., Mauchauffe, M., Le Coniat, M., et al. (1995) The t(12;21) of Acute Lymphoblastic Leukemia Results in a tel-AML1 Gene Fusion. Blood, 85, 3662-3670. https://doi.org/10.1182/blood.V85.12.3662.bloodjournal85123662

[5] Nucifora, G., Birn, D.J., Erickson, P., et al. (1993) Detection of DNA Rearrangements in the AML1 and ETO Loci and of an AML1/ETO Fusion mRNA in Patients with $\mathrm{t}(8 ; 21)$ Acute Myeloid Leukemia. Blood, 81, 883-888. https://doi.org/10.1182/blood.V81.4.883.bloodjournal814883

[6] Barr, F.G., Galili, N., Holick, J., et al. (1993) Rearrangement of the PAX3 Paired Box Gene in the Paediatric Solid Tumour Alveolar Rhabdomyosarcoma. Nature Genetics, 3, 113-117. https://doi.org/10.1038/ng0293-113

[7] Tomlins, S.A., Rhodes, D.R., Perner, S., et al. (2005) Recurrent Fusion of TMPRSS2 and ETS Transcription Factor Genes in Prostate Cancer. Science, 310, 644-648. https://doi.org/10.1126/science.1117679

[8] Kannan, K., Coarfa, C., Chao, P.W., et al. (2015) Recurrent BCAM-AKT2 Fusion Gene Leads to a Constitutively Activated AKT2 Fusion Kinase in High-Grade Serous Ovarian Carcinoma. Proceedings of the National Academy of Sciences of the United States of America, 112, E1272-E1277. https://doi.org/10.1073/pnas.1501735112

[9] Kim, D.S., Huh, J.W. and Kim, H.S. (2007) HYBRIDdb: A Database of Hybrid Genes in the Human Genome. BMC Genomics, 8, 128. https://doi.org/10.1186/1471-2164-8-128

[10] Wang, Y., Wu, N., Liu, J., et al. (2015) FusionCancer: A Database of Cancer Fusion Genes Derived from RNA-seq Data. Diagnostic Pathology, 10, 131. https://doi.org/10.1186/s13000-015-0310-4

[11] Prakash, T., Sharma, V.K., Adati, N., et al. (2010) Expression of Conjoined Genes: 
Another Mechanism for Gene Regulation in Eukaryotes. PLoS ONE, 5, e13284. https://doi.org/10.1371/journal.pone.0013284

[12] Novo, F.J., de Mendibil, I.O. and Vizmanos, J.L. (2007) TICdb: A Collection of Gene-Mapped Translocation Breakpoints in Cancer. BMC Genomics, 8, 33. https://doi.org/10.1186/1471-2164-8-33

[13] Kong, F., Zhu, J., Wu, J., et al. (2011) dbCRID: A Database of Chromosomal Rearrangements in Human Diseases. Nucleic Acids Research, 39, D895-D900. https://doi.org/10.1093/nar/gkq1038

[14] Suhre, K. and Claverie, J.M. (2004) FusionDB: A Database for In-Depth Analysis of Prokaryotic Gene Fusion Events. Nucleic Acids Research, 32, D273-D276. https://doi.org/10.1093/nar/gkh053

[15] Gorohovski, A., Tagore, S., Palande, V., et al. (2017) ChiTaRS-3.1-The Enhanced Chimeric Transcripts and RNA-seq Database Matched with Protein-Protein Interactions. Nucleic Acids Research, 45, D790-D795. https://doi.org/10.1093/nar/gkw1127

[16] Lee, M., Lee, K., Yu, N., et al. (2017) ChimerDB 3.0: An Enhanced Database for Fusion Genes from Cancer Transcriptome and Literature Data Mining. Nucleic Acids Research, 45, D784-D789.

[17] Mitelman, F. (2000) Recurrent Chromosome Aberrations in Cancer. Mutation Research, 462, 247-253. https://doi.org/10.1016/S1383-5742(00)00006-5

[18] Li, Z., Qin, F. and Li, H. (2018) Chimeric RNAs and Their Implications in Cancer. Current Opinion in Genetics and Development, 48, 36-43.

https://doi.org/10.1016/j.gde.2017.10.002

[19] Zhu, J. (1995) Analysis of Conditional Genetic Effects and Variance Components in Developmental Genetics. Genetics, 141, 1633-1639.

https://doi.org/10.1093/genetics/141.4.1633

[20] Aurias, A., Rimbaut, C., Buffe, D., et al. (1984) Translocation Involving Chromosome 22 in Ewing's Sarcoma. A Cytogenetic Study of Four Fresh Tumors. Cancer Genetics and Cytogenetics, 12, 21-25. https://doi.org/10.1016/0165-4608(84)90003-7

[21] Quintas-Cardama, A. and Cortes, J. (2009) Molecular Biology of Bcr-abl1-Positive Chronic Myeloid Leukemia. Blood, 113, 1619-1630.

https://doi.org/10.1182/blood-2008-03-144790

[22] Qin, F., Shi, X. and Li, H. (2020) Knockdown of Chimeric RNA by RNAi. Methods in Molecular Biology, 2079, 143-154. https://doi.org/10.1007/978-1-4939-9904-0 11

[23] Kannan, K., Wang, L., Wang, J., et al. (2011) Recurrent Chimeric RNAs Enriched in Human Prostate Cancer Identified by Deep Sequencing. Proceedings of the National Academy of Sciences of the United States of America, 108, 9172-9177. https://doi.org/10.1073/pnas.1100489108

[24] Nacu, S., Yuan, W., Kan, Z., et al. (2011) Deep RNA Sequencing Analysis of Read through Gene Fusions in Human Prostate Adenocarcinoma and Reference Samples. BMC Medical Genomics, 4, 11. https://doi.org/10.1186/1755-8794-4-11

[25] Yuan, C., Han, Y., Zellmer, L., et al. (2017) It Is Imperative to Establish a Pellucid Definition of Chimeric RNA and to Clear Up a Lot of Confusion in the Relevant Research. International Journal of Molecular Sciences, 18, 714. https://doi.org/10.3390/ijms18040714

[26] Qin, F., Song, Y., Zhang, Y., et al. (2016) Role of CTCF in Regulating SLC45A3-ELK4 Chimeric RNA. PLoS ONE, 11, e150382. 
https://doi.org/10.1371/journal.pone.0150382

[27] Rickman, D.S., Pflueger, D., Moss, B., et al. (2009) SLC45A3-ELK4 Is a Novel and Frequent Erythroblast Transformation-Specific Fusion Transcript in Prostate Cancer. Cancer Research, 69, 2734-2738. https://doi.org/10.1158/0008-5472.CAN-08-4926

[28] Kumar-Sinha, C., Kalyana-Sundaram, S. and Chinnaiyan, A.M. (2012) SLC45A3-ELK4 Chimera in Prostate Cancer: Spotlight on cis-Splicing. Cancer Discovery, 2, 582-585. https://doi.org/10.1158/2159-8290.CD-12-0212

[29] Zhang, Y., Gong, M., Yuan, H., et al. (2012) Chimeric Transcript Generated by cis-Splicing of Adjacent Genes Regulates Prostate Cancer Cell Proliferation. Cancer Discovery, 2, 598-607. https://doi.org/10.1158/2159-8290.CD-12-0042

[30] Milhausen, M., Nelson, R.G., Sather, S., et al. (1984) Identification of a Small RNA Containing the Trypanosome Spliced Leader: A Donor of Shared 5' Sequences of Trypanosomatid mRNAs? Cell, 38, 721-729.

https://doi.org/10.1016/0092-8674(84)90267-8

[31] Koller, B., Fromm, H., Galun, E., et al. (1987) Evidence for in Vivo Trans Splicing of pre-mRNAs in Tobacco Chloroplasts. Cell, 48, 111-119.

https://doi.org/10.1016/0092-8674(87)90361-8

[32] Kuck, U., Choquet, Y., Schneider, M., et al. (1987) Structural and Transcription Analysis of Two Homologous Genes for the P700 Chlorophyll a-Apoproteins in Chlamydomonas reinhardii: Evidence for in Vivo Trans-Splicing. The EMBO Journal, 6, 2185-2195. https://doi.org/10.1002/j.1460-2075.1987.tb02489.x

[33] Chwalenia, K., Facemire, L. and Li, H. (2017) Chimeric RNAs in Cancer and Normal Physiology. Wiley Interdisciplinary Reviews. $R N A, 8$, e1427. https://doi.org/10.1002/wrna.1427

[34] Greger, L., Su, J., Rung, J., et al. (2014) Tandem RNA Chimeras Contribute to Transcriptome Diversity in Human Population and Are Associated with Intronic Genetic Variants. PLoS ONE, 9, e104567. https://doi.org/10.1371/journal.pone.0104567

[35] Li, H., Wang, J., Mor, G., et al. (2008) A Neoplastic Gene Fusion Mimics Trans-Splicing of RNAs in Normal Human Cells. Science, 321, 1357-1361.

https://doi.org/10.1126/science.1156725

[36] Caudevilla, C., Serra, D., Miliar, A., et al. (1998) Natural Trans-Splicing in Carnitine Octanoyltransferase pre-mRNAs in Rat Liver. Proceedings of the National Academy of Sciences of the United States of America, 95, 12185-12190. https://doi.org/10.1073/pnas.95.21.12185

[37] Lei, Q., Li, C., Zuo, Z., et al. (2016) Evolutionary Insights into RNA Trans-Splicing in Vertebrates. Genome Biology and Evolution, 8, 562-577.

https://doi.org/10.1093/gbe/evw025

[38] Muddathir, A.M., Kordofani, A.A. and Fadl-Elmula, I.M. (2013) Frequency of BCR-ABL Fusion Transcripts in Sudanese Patients with Chronic Myeloid Leukemia Using Real-Time Reverse Transcription-Polymerase Chain Reaction. Saudi Medical Journal, 34, 29-33.

[39] Soda, M., Choi, Y.L., Enomoto, M., et al. (2007) Identification of the Transforming EML4-ALK Fusion Gene in Non-Small-Cell Lung Cancer. Nature, 448, 561-566. https://doi.org/10.1038/nature05945

[40] Yang, Y.Z., Pan, L.K. and An, G.Y. (2012) Biological Characteristics of EML4-ALK Fusion Gene in Non-Small Cell Lung Cancer and Its Treatment. Cancer, 32, 
466-470

[41] Wang, L.J. and Guo, Q.S. (2014) EML4-ALK Fusion Gene: A New Clinical Target for Advanced Non-Small Cell Lung Cancer. Chin J Lung Dis, No. 1, 108-111.

[42] Guo, Q. and Yu, Y.H. (2014) Expression and Significance of TMPRSS2-ERG Fusion Gene and ERG Protein in Prostate Cancer. Journal of Clinical and Experimental Pathology, No. 10, 1099-1103.

[43] Iyer, M.K., Niknafs, Y.S., Malik, R., et al. (2015) The Landscape of Long Noncoding RNAs in the Human Transcriptome. Nature Genetics, 47, 199-208. https://doi.org/10.1038/ng.3192

[44] Qin, F., Zhang, Y., Liu, J., et al. (2017) SLC45A3-ELK4 Functions as a Long Non-Coding Chimeric RNA. Cancer Letters, 404, 53-61. https://doi.org/10.1016/j.canlet.2017.07.007

[45] Maher, C.A., Kumar-Sinha, C., Cao, X., et al. (2009) Transcriptome Sequencing to Detect Gene Fusions in Cancer. Nature, 458, 97-101. https://doi.org/10.1038/nature07638

[46] Frenkel-Morgenstern, M., Lacroix, V., Ezkurdia, I., et al. (2012) Chimeras Taking Shape: Potential Functions of Proteins Encoded by Chimeric RNA Transcripts. Genome Research, 22, 1231-1242. https://doi.org/10.1101/gr.130062.111

[47] Babiceanu, M., Qin, F., Xie, Z., et al. (2016) Recurrent Chimeric Fusion RNAs in Non-Cancer Tissues and Cells. Nucleic Acids Research, 44, 2859-2872. https://doi.org/10.1093/nar/gkw032

[48] Wong, S. and Witte, O.N. (2004) The BCR-ABL Story: Bench to Bedside and Back. Annual Review of Immunology, 22, 247-306. https://doi.org/10.1146/annurev.immunol.22.012703.104753

[49] Zhang, H., Lin, W., Kannan, K., et al. (2013) Aberrant Chimeric RNA GOLM1-MAK10 Encoding a Secreted Fusion Protein as a Molecular Signature for Human Esophageal Squamous Cell Carcinoma. Oncotarget, 4, 2135-2143. https://doi.org/10.18632/oncotarget.1465

[50] Tang, Y. (2019) Analysis of Chimeric RNA in Infant Brain Tissue and Function Study of DUS4L-BCAP29. Zhengzhou University, Zhengzhou.

[51] Ke, X.R., Xiong, X., Lin, Y.S., et al. (2019) Research and Transformation of Exosome Tumor Vaccine. Wuhan, 1.

[52] Shtivelman, E., Lifshitz, B., Gale, R.P., et al. (1985) Fused Transcript of abl and bcr Genes in Chronic Myelogenous Leukaemia. Nature, 315, 550-554. https://doi.org/10.1038/315550a0

[53] Sattler, M. and Griffin, J.D. (2001) Mechanisms of Transformation by the BCR/ABL Oncogene. International Journal of Hematology, 73, 278-291. https://doi.org/10.1007/BF02981952

[54] Mitelman, F., Johansson, B. and Mertens, F. (2007) The Impact of Translocations and Gene Fusions on Cancer Causation. Nature Reviews Cancer, 7, 233-245. https://doi.org/10.1038/nrc2091

[55] Wu, P., Yang, S., Singh, S., et al. (2018) The Landscape and Implications of Chimeric RNAs in Cervical Cancer. EBioMedicine, 37, 158-167.

https://doi.org/10.1016/j.ebiom.2018.10.059

[56] Li, B.J., Zhu, Z.H., Wang, J.Y., et al. (2014) Expression Correlation of Ki67 to P53, VEGF, and C-erbB-2 Genes in Breast Cancer and Their Clinical Significances. Chinese Journal of Cancer, 23, 1176-1179.

[57] Ma, L. and Zhang, T.T. (2018) Application of Chimeric Gene Examples to Expand 
the Teaching of Genetic Chromosome Aberrations. Genetic, 40, 1129-1136.

[58] Elfman, J. and Li, H. (2018) Chimeric RNA in Cancer and Stem Cell Differentiation. Stem Cells International, 2018, Article ID: 3178789.

https://doi.org/10.1155/2018/3178789

[59] Li, H., Wang, J., Ma, X., et al. (2009) Gene Fusions and RNA Trans-Splicing in Normal and Neoplastic Human Cells. Cell Cycle, 8, 218-222. https://doi.org/10.4161/cc.8.2.7358

[60] Yuan, H., Qin, F., Movassagh, M., et al. (2013) A Chimeric RNA Characteristic of Rhabdomyosarcoma in Normal Myogenesis Process. Cancer Discovery, 3, 1394-1403. https://doi.org/10.1158/2159-8290.CD-13-0186

[61] Tang, Y., Qin, F., Liu, A., et al. (2017) Recurrent Fusion RNA DUS4L-BCAP29 in Non-Cancer Human Tissues and Cells. Oncotarget, 8, 31415-31423. https://doi.org/10.18632/oncotarget.16329

[62] Tang, Y., Ma, S., Wang, X., et al. (2019) Identification of Chimeric RNAs in Human Infant Brains and Their Implications in Neural Differentiation. The International Journal of Biochemistry \& Cell Biology, 111, 19-26.

https://doi.org/10.1016/j.biocel.2019.03.012

[63] Rickman, D.S., Beltran, H., Demichelis, F., et al. (2017) Biology and Evolution of Poorly Differentiated Neuroendocrine Tumors. Nature Medicine, 23, 1-10. https://doi.org/10.1038/nm.4341

[64] van der Kouwe, E. and Staber, P.B. (2019) RUNX1-ETO: Attacking the Epigenome for Genomic Instable Leukemia. International Journal of Molecular Sciences, 20, 350. https://doi.org/10.3390/ijms20020350

[65] Anetta, P., Anna, P., Salam, A.A., et al. (2019) RUNX1-ETO Depletion in t(8;21) AML Leads to C/EBP $\alpha$ - and AP-1-Mediated Alterations in Enhancer-Promoter Interaction. Cell Reports, 28, 3022-3031.e1-e7. https://doi.org/10.1016/j.celrep.2019.08.040 